\title{
Dynamic Contrast-Enhanced MRI of Ocular Biotransport in Normal and Hypertensive Eyes
}

\author{
Kevin C. Chan, Qing-ling Fu, Kwok-fai So, and Ed X. Wu
}

\begin{abstract}
This study aims to employ in vivo dynamic contrast-enhanced magnetic resonance imaging (DCE-MRI) to evaluate the ocular transport following an induction of ocular hypertension in a rat model of chronic glaucoma. Upon systemic administration of Gd-DTPA solution, T1-weighted signal increase was observed in the vitreous body of the glaucomatous eye but not the control eye. This increase occurred earlier in the anterior vitreous body than the preretinal vitreous. Further, there was an earlier Gd-DTPA transport into the anterior chamber in the majority of glaucomatous eyes. Our DCE-MRI findings revealed the leakage of Gd-DTPA at the aqueous-vitreous interface, which was likely resulted from increased permeability of blood-aqueous or aqueous-vitreous barrier. These may explain the sources of changing biochemical compositions in the chamber components, which may implicate the neurodegenerative processes in the glaucomatous visual components.
\end{abstract}

\section{INTRODUCTION}

$\mathrm{G}$ LAUCOMA is a neurodegenerative disease of the visual system characterized by an increase in intraocular pressure (IOP). It is the second major cause of blindness in the world [1]. While the level of IOP is governed by the dynamic balance between aqueous humor formation and drainage [2], the elevated IOP in primary open-angle glaucoma (POAG) is believed to arise due to an increased resistance to the outflow of aqueous humor from the eye [3]. Nevertheless, due to the lack of a non-invasive method to monitor the inner-depth of the eye without critically affecting the dynamic behavior of the aqueous outflow system, the aqueous outflow obstructions in glaucoma have not been well understood [4].

MRI allows inner-depth localization of dynamic ocular processes in vivo. Recent studies using the arterial spin labeling technique showed high basal blood flows in the ciliary body and retina of rat eyes [5]. In the ciliary body, the aqueous humor is produced primarily by active transport

Manuscript received April 7, 2008. This work was supported in part by the Hong Kong Research Grant Council and The University of Hong Kong CRCG grant

Kevin C. Chan is with the Laboratory of Biomedical Imaging and Signal Processing and the Department of Electrical and Electronic Engineering, The University of Hong Kong, Pokfulam, Hong Kong (e-mail: kevin_ccw@hkusua.hku.hk).

Qing-ling Fu and Kwok-fai So are with the Department of Anatomy and The State Key Laboratory of Brain and Cognitive Sciences, The University of Hong Kong, Pokfulam, Hong Kong (e-mail: qlfu@graduate.hku.hk and hrmaskf@hkucc.hku.hk).

Ed X. Wu is with the Laboratory of Biomedical Imaging and Signal Processing and the Department of Electrical and Electronic Engineering, The University of Hong Kong, Pokfulam, Hong Kong (corresponding author to provide phone: (852) 2819-9714; fax: (852) 2819-9711; e-mail: ewu@eee.hku.hk). across epithelial cells lining the surface of the ciliary processes [6], and is drained away from the venous plexus of the limbus via a circumferential Schlemm's canal into multiple radial veins located within the episclera [2]. This conventional route of aqueous humor outflow [7] taken by plasma-derived proteins has been traced using dynamic contrast-enhanced magnetic resonance imaging (DCE-MRI) [8], by demonstrating the entrance of an exogenous MR contrast agent Gd-DTPA from the bloodstream into the ciliary body stroma, and its exit via the iris root [9]. This potentiated the study of changes in aqueous flow in the rat model of chronic glaucoma using DCE-MRI.

Despite the relatively small molecular mass of $\mathrm{Gd}$ DTPA (938 Da), in normal intact eyes, the blood-retinal barrier and aqueous-vitreous barrier are impermeable to $\mathrm{Gd}$ DTPA [10, 11]. DCE-MRI has been used to examine the pathology that altered the permeability of blood-aqueous barrier [9, 12] and blood-retinal barrier [11, 13] in both human and animal eyes. On the basis of the previous findings about the potential roles of blood-ocular [14-16] and aqueous-vitreous barrier integrity [17-19] in the pathogenesis of POAG, this study aims to employ high resolution DCEMRI to understand the aqueous humor dynamics following an induction of ocular hypertension in a rat model of chronic glaucoma. We hypothesized that systemic administration of Gd-DTPA into our glaucoma model would detect whether leakage existed at the aqueous-vitreous interface giving rise to a progressive signal enhancement in the anterior vitreous body of the glaucomatous eye. Further, we attempted to monitor the transport of Gd-DTPA into the anterior chamber of our glaucoma model. Such transport phenomena may help understand the sources of biochemicals present in the chamber components of the glaucomatous eye, which may have participated in the neurodegenerative processes in the retina and the optic nerve $[20,21]$.

\section{MATERIALS AND METHODS}

\section{A. Animal Preparation}

Sprague-Dawley female rats (250-280 g, 3 months old, $\mathrm{N}=11$ ) were divided into two groups and were induced for ocular hypertension unilaterally in the right eye by photocoagulation of episcleral veins and the limbal veins on the surface of the eyeball using an argon laser. DCE-MRI was performed at Day 5 (Group 1, n=5) and Week 8 (Group $2, n=6)$ after laser treatment to maintain a consistent IOP elevation by about 1.6 times above the normal level. This technique was modified from the method by WoldeMussie et 
al [22] and has been adopted in our laboratory for the study of retinal degeneration and therapeutic effects on retinal functions [20, 21, 23, 24]. After each procedure, antibiotic ointment was applied topically to the eye surface.

\section{B. MRI Protocols}

All MRI measurements were acquired on a $7 \mathrm{~T}$ Bruker scanner using a birdcage transmit-only RF coil in combination with an actively decoupled receive-only quadrature surface coil. Under inhaled isoflurane anaesthesia (3\% induction and $1.5 \%$ maintenance), the animal was kept warm under circulating water at $37{ }^{\circ} \mathrm{C}$. A saline phantom was inserted beside the rat for signal intensity normalization during post-processing. Five days (Group 1) and 8 weeks (Group 2) after laser treatment, Gd-DTPA (Magnevist) was applied intraperitoneally at $3 \mathrm{mmol} / \mathrm{kg}$, and 2D fast spin echo (FSE) T1-weighted imaging (T1WI) was acquired with fat suppression, TR/TE $=320 / 8.9 \mathrm{~ms}$, echo train length $=4$, $\mathrm{NEX}=26$, slice thickness $=1 \mathrm{~mm}, \mathrm{FOV}=3.2 \times 3.2 \mathrm{~cm}^{2}$ and voxel resolution $=82.3 \times 82.3 \mu \mathrm{m}^{2}$. Acquisition time was 10 minutes for each time point, and T1WI was applied before and 10 to 80 minutes after injection. Throughout the experiments, the left eye served as a control. Between 0 and 10 minutes after injection, T1WI was also acquired in Group 1 with the same T1WI protocol above but with NEX $=5$ and acquisition time $=2 \mathrm{~min}$ for each time point to monitor the enhanced pattern immediately after injection.

\section{Data Analysis}

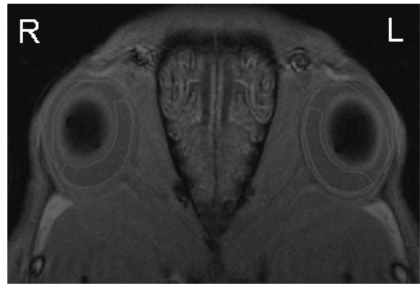

Before injection

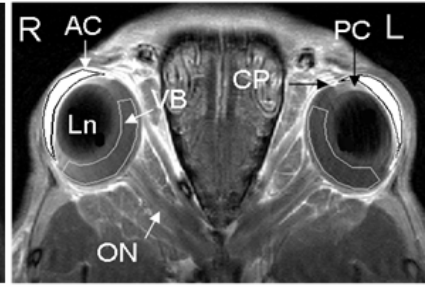

60 mins after injection
Fig. 1. Illustration of the typical ROI definitions of the anterior chamber (AC) and vitreous body (VB) to quantify the DCE-MRI measurements. The lens $(\mathrm{Ln})$ and optic nerves $(\mathrm{ON})$ could be observed as hypointense signals in both pre- (left) and post- (right) systemic Gd-DTPA injection images, while the hyperintense ciliary processes could be found between the posterior chamber (PC) and VB after systemic Gd-DTPA injection (right).

In the $2 \mathrm{D}$ slice that centrally cut through the eyeball and optic nerve head, regions of interest (ROIs) were drawn manually on the whole anterior chamber, and on the vitreous body covering a distance of $3.5 \mathrm{~mm}$ on each side from the optic nerve head using ImageJ v1.38 (Wayne Rasband, NIH, USA) as shown in Figure 1. Each value was calibrated to the nearby phantom containing saline solution to avoid the effect of any MRI system sensitivity drift. A quantitative measure of the Gd-DTPA entry was obtained from the enhancement in the MR signal intensity defined as $E=\left[S(t)-S_{0}\right] / S_{0}$, where $S(t)$ is the calibrated spin-echo signal intensity of the ROIs, and $\mathrm{S}_{\mathrm{o}}$ is the T1-weighted signal intensity of the same ROI before the MR tracer injection. Differences between mean values of the ROIs on both sides were compared using two-tailed paired t-tests, and the mean values along the time course were compared using ANOVA. Results were considered to be significantly different when $\mathrm{p}<0.05$.

\section{RESULTS}

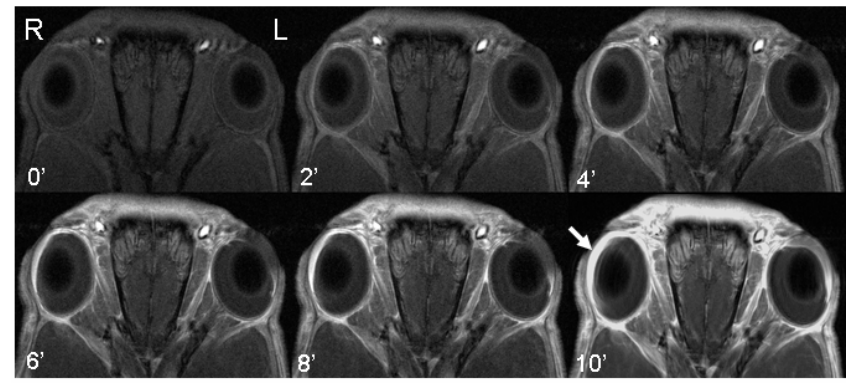

Fig. 2. Serial T1-weighted images (T1WIs) of a rat at Day 5 (Group 1) after glaucoma induction. Images were acquired within the first 10 minutes following systemic Gd-DTPA administration. Note that in 3 out of the 5 rats in the group, the intensity in the anterior chamber was greater for the glaucomatous eye (R) (arrow) than the control one (L) in the first 10 minutes after systemic Gd-DTPA administration.

Figures 2 and 3 show the serial T1WIs of the glaucomatous and control eyes after systemic Gd-DTPA administration. Figure 4 shows the mean percent signal enhancement, E, as a function of the time for the vitreous body in both eyes. In the control eye, there was an immediate enhancement of the ciliary processes, iris and chorioretina in all scanned rats after Gd-DTPA administration (Figure 2). The anterior chamber enhanced substantially over the first 40-60 minutes after Gd-DTPA administration (ANOVA, $\mathrm{p}<0.01$ ), while the vitreous and lens did not enhance at any time (ANOVA, p>0.05) (Figure 3). These patterns of T1weighted signal enhancements generally followed as previously described [8-10, 25] and were of consistent or better spatial and temporal resolutions.

Similar patterns appeared in the glaucomatous eye. However, the vitreous body enhanced progressively from 10 minutes post-administration in both groups after glaucoma induction (Figures 3 and 4) (ANOVA, p $<0.01$ ). Significant difference was observed between the glaucomatous and the control eyes in Group 2 (paired t-test, $\mathrm{p}<0.05$ ), and was marginally observed in Group 1 (paired t-test, $\mathrm{p}<0.07$ for the later 2 time points). No apparent difference was observed between the two groups on the signal changes in the glaucomatous eyes (unpaired t-test, $\mathrm{p}>0.05$ ). Qualitative inspection of the MR images of both groups suggested that Gd-DTPA leaked at the aqueous-vitreous interface and diffused into the vitreous body from the anterior parts of the glaucomatous eye in all rats, while no apparent signal increase was observed in the same location in the control eyes (Figure 3). Note that in 3 out of the 5 rats in Group 1, the intensity in the anterior chamber is greater for the glaucomatous eye than the control one in the first 10 minutes after Gd-DTPA administration (Figure 2). For Group 2, 4 out of 6 rats had their signal intensities higher in the glaucomatous anterior chamber than the control throughout the experiment. 

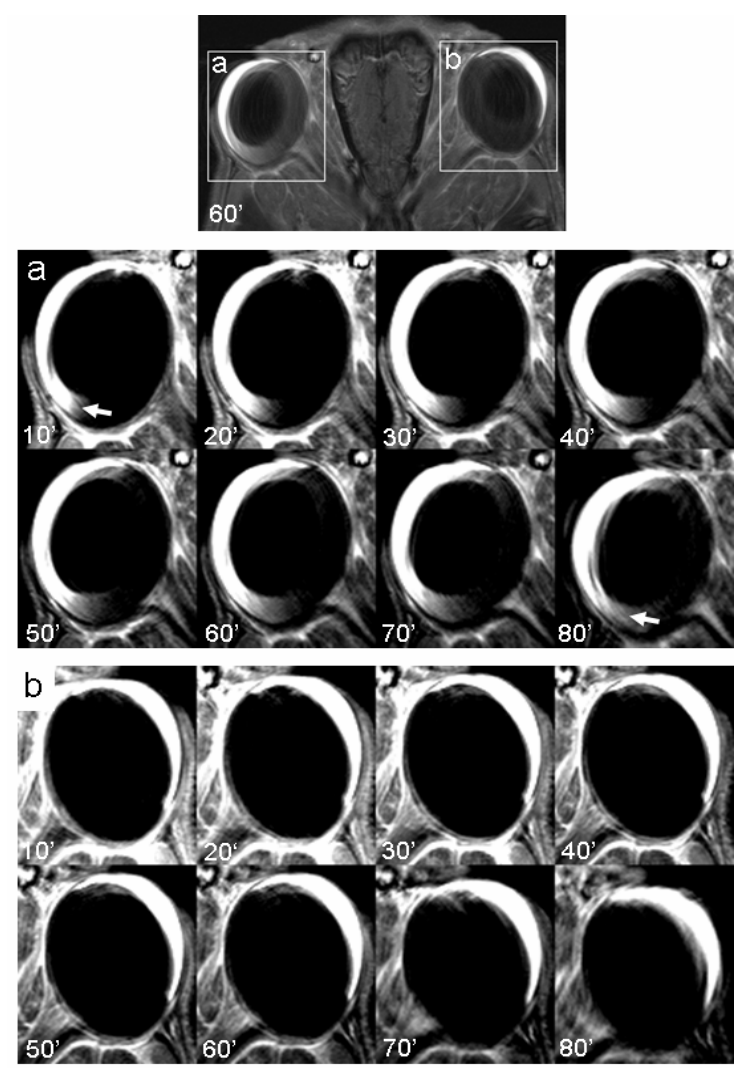

Fig. 3. Windowed and zoomed serial T1WIs of the Week 8 (Group 2) glaucomatous (a) and control (b) eyeballs 10 to 80 minutes following systemic Gd-DTPA administration. The corresponding un-windowed image at 80 minutes post-administration was also shown on top to illustrate the regions of interest for zooming. Different extents of leakiness from the aqueous-vitreous interface (arrows) into the vitreous body were visible in the glaucomatous eye but not the control eye as time went by.

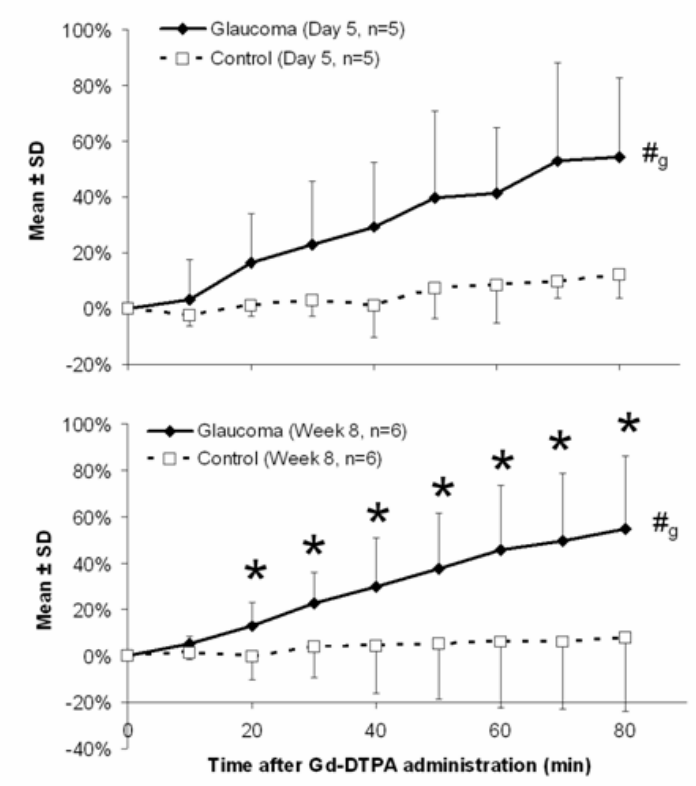

Fig. 4. Time course of $\mathrm{T} 1 \mathrm{~W}$ signal enhancement of the vitreous body of both eyes before and at 10-80 minutes following Gd-DTPA administration into both groups. The vitreous body did not enhance in the control eye, yet there was a progressive increase in signal intensity in the glaucomatous eye. (ANOVA across timeline in glaucomatous (g) and control (c) eyes with

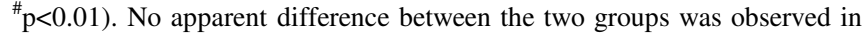
the signal changes in the glaucomatous or control eye.

\section{Discussions}

In the present study we characterized the aqueous humor dynamics in a rat model of chronic glaucoma. Obstruction of aqueous humor outflow is the primary mechanism of pressure elevation in the current glaucoma model [26] analogous to the pathogenesis of human POAG [27]. Our DCE-MRI results showed an alteration of the typical aqueous outflow in the glaucomatous eye that might explain the changing biochemical compositions in the anterior chamber and vitreous body $[15,16,19]$.

The aqueous humor proteins have been identified as suspected participants in the obstruction of the aqueous humor outflow network from the eye resulting in elevated IOP in POAG [27]. Protein concentration in the anterior chamber of normal eyes has been identified to be determined by the rate of protein entry into the aqueous humor, the removal rate by bulk flow of aqueous humor, and the anterior chamber volume [28]. Our results of increased GdDTPA level in the anterior chamber appeared to correlate with the breakdown of blood-aqueous barrier in various glaucoma which resulted in an increase in the aqueous protein level upon the presence of cytokines and other inflammatory mediators in the aqueous humor [9, 12] including vascular endothelial growth factor [16] and endothelin-1 [15].

On the other hand, the earlier T1-weighted signal increase in the anterior vitreous body indicated the leakage of GdDTPA at the aqueous-vitreous interface upon aqueous outflow obstruction. A recent study had commented on the impact of IOP on the induction of the hydraulic flow permeating through the vitreous body [18]. When the IOP was increased in the current glaucoma model, it was likely that this anteriorly directed fluid movement across the aqueous-vitreous interface would be reduced [29]. In addition, in response to prolonged IOP elevation, the ciliary epithelium which prevents the aqueous humor proteins from entering the posterior chamber by its tight junctions [27], has been shown to undergo atrophy in canine [30]. Iris atrophy was also observed in a transgenic glaucoma model [31]. The reduced limitation of back-diffusion of solutes might attribute to the posterior transport of Gd-DTPA from the anterior chamber to the vitreous body. Note that the pattern of leakage of proteins from the blood supply in the ciliary body/iris complex has been postulated in experimental newborn rats, when the tight junctions of the blood-ocular barriers were underdeveloped [32].

In one of the glaucomatous eyes in Group 2, a faster leakage into the vitreous body was observed compared to the other diseased eyes of the same group, while a prominent lens depression was also noted (Figure 5). Upon perfusion of the anterior chamber at elevated pressures, the iris and the detached portion of the ciliary body were pushed backwards [17]. A significant increase in the number of giant vacuoles was also found in the endothelium of several blood vessels in the rat ciliary body and the iris root [7]. These might also 
contribute to the compromise of the aqueous-vitreous barrier integrity, allowing Gd-DTPA to pass into the vitreous body. Our results potentially explained the routes for the leakage of serum proteins as well as the release of proteolytic enzymes into the rat vitreous body upon chronic intraocular hypertension [19]. DCE-MRI was also performed at Week 18 on three of the Group 2 animals scanned, and similar pattern of Gd-DTPA leakage was observed as in Groups 1 and 2 (data not shown). No statistical difference was observed in the signal changes in the glaucomatous eyes between two groups, which was in agreement with the fact that a consistent IOP elevation of 1.6 times above normal was maintained in our model across the 8-week experimental period [21]. Further studies may employ multi-compartment modeling for more comprehensive kinetics analyses in the future.

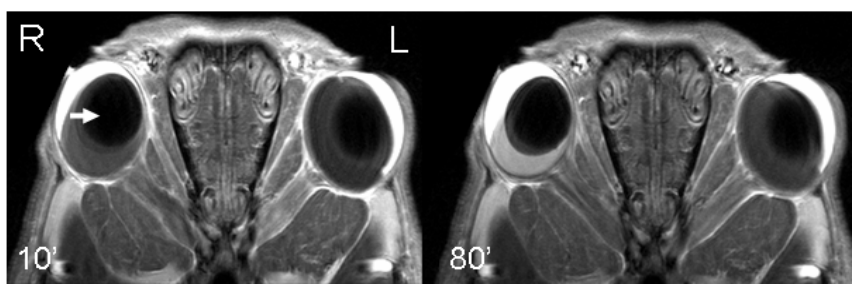

Fig. 5. Leakage of Gd-DTPA into the vitreous body appeared faster in one of the 6 glaucomatous eyes in Group 2. A prominent lens depression was observed at the same time along the direction pointed by the arrow.

\section{CONCLUSIONS}

The results presented here constitute the first report in assessing the leakage at the aqueous-vitreous interface upon ocular hypertension by DCE-MRI. A progressive T1weighed signal increase was observed in the vitreous body of the glaucomatous eye but not the control eye, while an earlier Gd-DTPA transport was found in the anterior chamber of the glaucomatous eye. The authors demonstrated the values of MRI in providing a means for direct noninvasive visualization of physiological kinetics in the glaucomatous chamber components in vivo.

\section{REFERENCES}

[1] H. A. Quigley and A. T. Broman, "The number of people with glaucoma worldwide in 2010 and 2020," Br J Ophthalmol, vol. 90, pp. 262-7, Mar 2006

[2] J. C. Morrison, F. W. Fraunfelder, S. T. Milne, and C. G. Moore, "Limbal microvasculature of the rat eye," Invest Ophthalmol Vis Sci, vol. 36, pp. 751-6, Mar 1995.

[3] M. Johnson, "'What controls aqueous humour outflow resistance?'" Exp Eye Res, vol. 82, pp. 545-57, Apr 2006.

[4] P. L. Kaufman, "Nitric-oxide synthase and neurodegeneration/neuroprotection," Proc Natl Acad Sci U S A, vol. 96, pp. 9455-6, Aug 171999.

[5] Y. Li, H. Cheng, and T. Q. Duong, "Blood-flow magnetic resonance imaging of the retina," Neuroimage, vol. 39, pp. 1744-51, Feb 152008.

[6] T. J. Jacob and M. M. Civan, "Role of ion channels in aqueous humor formation," Am J Physiol, vol. 271, pp. C703-20, Sep 1996.

[7] P. G. McMenamin and M. J. al-Shakarchi, "The effect of various levels of intraocular pressure on the rat aqueous outflow system," J Anat, vol. 162, pp. 6782, Feb 1989.

[8] R. J. Bert, S. D. Caruthers, H. Jara, J. Krejza, E. R. Melhem, N. H. Kolodny, S. Patz, and T. F. Freddo, "Demonstration of an anterior diffusional pathway for solutes in the normal human eye with high spatial resolution contrast-enhanced dynamic MR imaging," Invest Ophthalmol Vis Sci, vol. 47, pp. 5153-62, Dec 2006.
[9] N. H. Kolodny, S. T. Goode, W. Ryan, and T. F. Freddo, "Evaluation of therapeutic effectiveness using MR imaging in a rabbit model of anterior uveitis," Exp Eye Res, vol. 74, pp. 483-91, Apr 2002.

[10] N. H. Kolodny, T. F. Freddo, B. A. Lawrence, C. Suarez, and S. P. Bartels, "Contrast-enhanced magnetic resonance imaging confirmation of an anterio protein pathway in normal rabbit eyes," Invest Ophthalmol Vis Sci, vol. 37, pp. 1602-7, Jul 1996.

[11] C. A. Wilson, B. A. Berkowitz, H. Funatsu, D. C. Metrikin, D. W. Harrison, M K. Lam, and P. L. Sonkin, "Blood-retinal barrier breakdown following experimental retinal ischemia and reperfusion," Exp Eye Res, vol. 61, pp. 547-57, Nov 1995.

[12] T. F. Freddo, S. Patz, and Y. Arshanskiy, "Pilocarpine's effects on the bloodaqueous barrier of the human eye as assessed by high-resolution, contrast magnetic resonance imaging," Exp Eye Res, vol. 82, pp. 458-64, Mar 2006.

[13] B. A. Berkowitz, R. Roberts, H. Luan, J. Peysakhov, X. Mao, and K. A. Thomas, "Dynamic contrast-enhanced MRI measurements of passive permeability through blood retinal barrier in diabetic rats," Invest Ophthalmol Vis Sci, vol. 45, pp. 23918, Jul 2004.

[14] N. I. Kuryshova, M. I. Vinetskaia, V. P. Erichev, V. P. Artamonov, and A. P. Uspenskaia, "[Permeability of blood-aqueous humor barrier in primary open-angle glaucoma]," Vestn Oftalmol, vol. 114, pp. 10-3, Jan-Feb 1998.

[15] G. Prasanna, C. Hulet, D. Desai, R. R. Krishnamoorthy, S. Narayan, A. M. Brun, A. M. Suburo, and T. Yorio, "Effect of elevated intraocular pressure on endothelin-1 in a rat model of glaucoma," Pharmacol Res, vol. 51, pp. 41-50, Jan 2005.

[16] D. N. Hu, R. Ritch, J. Liebmann, Y. Liu, B. Cheng, and M. S. Hu, "Vascular endothelial growth factor is increased in aqueous humor of glaucomatous eyes," $J$ Glaucoma, vol. 11, pp. 406-10, Oct 2002.

[17] F. J. Carreras, D. Porcel, and F. Gonzalez-Caballero, "Expanding forces in aqueous outflow pathways of a nonaccommodating mammal: an approach via comparative dynamic morphology," Comp Biochem Physiol A Physiol, vol. 117, pp. 197-209, Jun 1997.

[18] J. Xu, J. J. Heys, V. H. Barocas, and T. W. Randolph, "Permeability and diffusion in vitreous humor: implications for drug delivery," Pharm Res, vol. 17, pp. 664-9, Jun 2000.

[19] H. Levkovitch-Verbin, K. R. Martin, H. A. Quigley, L. A. Baumrind, M. E. Pease, and D. Valenta, "Measurement of amino acid levels in the vitreous humor of rats after chronic intraocular pressure elevation or optic nerve transection," $J$ Glaucoma, vol. 11, pp. 396-405, Oct 2002.

[20] R. S. Li, B. Y. Chen, D. K. Tay, H. H. Chan, M. L. Pu, and K. F. So, "Melanopsin-expressing retinal ganglion cells are more injury-resistant in a chronic ocular hypertension model," Invest Ophthalmol Vis Sci, vol. 47, pp. 2951-8, Jul 2006.

[21] R. S. Li, D. K. Tay, H. H. Chan, and K. F. So, "Changes of retinal functions following the induction of ocular hypertension in rats using argon laser photocoagulation," Clin Experiment Ophthalmol, vol. 34, pp. 575-83, Aug 2006.

[22] E. WoldeMussie, G. Ruiz, M. Wijono, and L. A. Wheeler, "Neuroprotection of retinal ganglion cells by brimonidine in rats with laser-induced chronic ocular hypertension," Invest Ophthalmol Vis Sci, vol. 42, pp. 2849-55, Nov 2001.

[23] K. C. Chan, Q. L. Fu, E. S. Hui, K. F. So, and E. X. Wu, "Evaluation of the retina and optic nerve in a rat model of chronic glaucoma using in vivo manganeseenhanced magnetic resonance imaging," Neuroimage, Jan 172008.

[24] Q. L. Fu, B. Hu, W. Wu, R. B. Pepinsky, S. Mi, and K. F. So, "Blocking LINGO1 Function Promotes Retinal Ganglion Cell Survival Following Ocular Hypertension and Optic Nerve Transection," Invest Ophthalmol Vis Sci, vol. 49 , pp. 975-85, Mar 2008.

[25] N. Alikacem, T. Yoshizawa, K. D. Nelson, and C. A. Wilson, "Quantitative MR imaging study of intravitreal sustained release of VEGF in rabbits," Invest Ophthalmol Vis Sci, vol. 41, pp. 1561-9, May 2000.

[26] L. Jia, W. O. Cepurna, E. C. Johnson, and J. C. Morrison, "Patterns of intraocular pressure elevation after aqueous humor outflow obstruction in rats," Invest Ophthalmol Vis Sci, vol. 41, pp. 1380-5, May 2000.

[27] M. F. Barsotti, S. P. Bartels, T. F. Freddo, and R. D. Kamm, "The source of protein in the aqueous humor of the normal monkey eye," Invest Ophthalmol Vis Sci, vol. 33, pp. 581-95, Mar 1992.

[28] D. L. Murray and S. P. Bartels, "The relationship between aqueous humor flow and anterior chamber protein concentration in rabbits," Invest Ophthalmol Vis Sci, vol. 34, pp. 370-6, Feb 1993.

[29] Y. Sugiura and M. Araie, "Effects of intraocular pressure change on movement of FITC-dextran across vitreous-aqueous interface," Jpn J Ophthalmol, vol. 33, pp. 441-50, 1989.

[30] R. Smith, R. Peiffer, and B. Wilcock, "Some aspects of the pathology of canine glaucoma," Prog. Vet. Comp. Ophthalmol, vol. 3, pp. 16-28, 1993.

[31] S. W. John, R. S. Smith, O. V. Savinova, N. L. Hawes, B. Chang, D. Turnbull, M Davisson, T. H. Roderick, and J. R. Heckenlively, "Essential iris atrophy, pigment dispersion, and glaucoma in DBA/2J mice," Invest Ophthalmol Vis Sci, vol. 39, pp. 951-62, May 1998.

[32] B. A. Berkowitz, K. A. Roberto, and J. S. Penn, "The vitreous protein concentration is increased prior to neovascularization in experimental ROP," Curr Eye Res, vol. 17, pp. 218-21, Feb 1998 